\title{
NADH dehydrogenase complex I is overexpressed in incipient metastatic murine colon cancer cells
}

\author{
JOANA MARQUEZ ${ }^{1}$, IRINA KRATCHMAROVA ${ }^{2}$, VYACHESLAV AKIMOV ${ }^{2}$, FERNANDO UNDA ${ }^{1}$, \\ GASKON IBARRETXE ${ }^{1}$, ARIANE SCHAUB CLERIGUÉ ${ }^{3}$, \\ NEREA OSINALDE ${ }^{4}$ and IKER BADIOLA ${ }^{1}$
}

\author{
${ }^{1}$ Department of Cell Biology and Histology, Faculty of Medicine and Nursing, University of Basque Country (UPV/EHU), \\ 48940 Leioa, Spain; ${ }^{2}$ Department of Biochemistry and Molecular Biology, University of Southern Denmark, \\ 5230 Odense M, Denmark; ${ }^{3}$ CIC bioGUNE, Bizkaia Technology Park, Derio, 48160 Bizkaia; \\ ${ }^{4}$ Department of Biochemistry and Molecular Biology, Faculty of Pharmacy, \\ UPV/EHU, 01006 Vitoria-Gasteiz, Spain
}

Received March 15, 2018; Accepted October 12, 2018

DOI: $10.3892 /$ or.2018.6892

\begin{abstract}
Colon cancer is one of the most frequently occurring types of cancers in the world. Primary tumours are treated very efficiently, but the metastatic cases are known to have severe outcomes. Therefore, the aim of the present study was to obtain a greater understanding of the transformation of primary colon cancer cells into metastatic phenotypes. Small changes in protein expression provoke the metastatic phenotype transformation. More sensitive methods to detect small variations are required. A murine colon cancer cell line with metastatic characteristics in a very early phase was created in order to investigate the first steps of transformation using a murine liver metastasis model. The protein expression patterns of metastatic and non-metastatic cells were compared using the stable isotope labelling by amino acids in cell culture method in combination with mass spectrometry. Quantitative proteomics data indicated that nicotinamide adenine dinucleotide hydride (NADH) dehydrogenase complex I was overexpressed in metastatic cells with respect to non-metastatic cells. Since the NADH dehydrogenase complex catalyses the oxidation of $\mathrm{NADH}$ to $\mathrm{NAD}^{+}$, the functionality of the complex was studied by measuring the amount of $\mathrm{NADH}$. The results revealed that
\end{abstract}

Correspondence to: Dr Nerea Osinalde, Department of Biochemistry and Molecular Biology, Faculty of Pharmacy, UPV/EHU, Unibertsitateko ibilbidea 7, 01006 Vitoria-Gasteiz, Spain

E-mail: nerea.osinalde@ehu.eus

Dr Iker Badiola, Department of Cell Biology and Histology, Faculty of Medicine and Nursing, University of Basque Country (UPV/EHU), B ${ }^{\circ}$ Sarriena s/n, 48940 Leioa, Spain

E-mail: iker.badiola@.ehu.eus

Key words: colon cancer, liver metastasis, nicotinamide adenine dinucleotide hydride dehydrogenase complex I, stable isotope labelling of amino acids in cell culture, Warburg effect metastatic cells accumulate more NADH and reactive oxygen species. In addition, the mitochondrial membrane potential of metastatic cells was lower than that of non-metastatic cells, indicating that the activity of NADH dehydrogenase and the mitochondrial oxidative chain were decreased in metastatic cells. During the incipient transformation of primary cancer cells, NADH dehydrogenase complex I was overexpressed but then became inactive due to the Warburg effect, which inhibits mitochondrial activity. In the first step of transformation, the high energy demand required in an adverse environment is fulfilled by overexpressing components of the respiratory chain, a fact that should be considered for future anti-metastatic therapies.

\section{Introduction}

Colorectal cancer (CRC) is one of the most common types of cancers and the second leading cause of cancer-associated mortalities among men and women combined (1). The prevalence of CRC has been gradually increasing over the last decade, and although the causes are not fully understood, certain factors such as lifestyle-associated stress, an unhealthy diet and environmental pollution are known to increase the risk of developing this disease (2).

Comparative studies between primary and metastatic tumour cells are preferentially performed using specific animal metastasis models, as Fidler (3) first employed to study melanoma. In this investigation, murine melanoma B16 cells were inoculated systemically into mice and, upon animal sacrifice, metastasized lung cells were collected. The metastatic cells collected in the lungs were injected into other mice 10 times. Consequently, during the repetition of the experiment, the cells obtained all of the molecular and phenotypic changes required to become metastatic cells. The same strategy has also been applied to study distinct metastatic processes, including breast to liver metastasis (4), melanoma to brain metastasis (5) and colorectal cancer to liver metastasis, using an orthotopic mouse model of the metastasis of 
human colon cancer to liver (6). As aforementioned, many of these investigations focused on deciphering the molecular events driving metastasis, generating the metastatic cell line by repeatedly inoculating the same cells into the animals until they completed all of the transformations required to be metastatic. However, it is important to consider that the intermediate steps that primary cancer cells undergo prior to the completion of the metastatic transformation may be essential to obtain a better understanding of the cellular, molecular and biological processes that result in metastasis. Thus, in order to elucidate the incipient transformations of the primary cells during their metastatic transition, the present study used a murine liver metastasis model in which liver metastasis was induced by intrasplenic injection of CRC cells. Fifteen days following the inoculation, cancer cells that remained in the spleen were considered to be non-metastatic, whereas cancer cells that had colonized the liver were considered to be metastatic cells. Unlike the Fidler experiment where the cells were collected following 10 inoculations to ensure that the collected cells were completely transformed, the present study performed a single cell inoculation and therefore collected cancer cells that exhibited marks of incipient transformations.

In the last few years, a number of large-scale comparative studies have been performed to identify discrete molecular patterns within primary and metastatic cancer cells by comparing their metabolism, DNA methylation, gene expression and microRNA expression pattern, among other characteristics (7-11). Considering the pivotal role of proteins in orchestrating the majority of all cellular functions, it is not surprising that the proteomes of primary and metastatic cancer cells have been extensively studied as well (10). Specifically, with the aim to elucidate the key factors involved in CRC malignancy, Tan et al (12) investigated the differences in protein expression observed between a primary and metastatic CRC cell line using 2-dimensional (2D) gel electrophoresis in combination with mass spectrometry (MS). This study identified 148 differentially expressed protein spots involved in a wide range of cellular functions (12).

Due to the numerous inherent drawbacks of 2D gel-based proteomic approaches for protein identification and quantification, various gel-free alternatives have been developed in the last decade. One of the most widely applied MS-based methods to simultaneously quantify changes in thousands of proteins with high accuracy and sensitivity is the so-called, stable isotope labelling of amino acids in cell culture (SILAC) method (13). SILAC is a metabolic labelling technique that consists of growing cells that are going to be compared in the presence of isotopically distinct versions of lysine and arginine. Upon complete labelling of their corresponding proteomes, differentially labelled cells are lysed, and the resulting protein extracts are equitably combined and processed in a single workflow. The greatest advantage of SILAC over other chemical-labelling methods, including isobaric tags for relative and absolute quantitation, tandem mass tags or isotope-coded affinity tag, is that samples are mixed at early stages of the sample process, and so sample loss and variability are significantly diminished, which leads to high reproducibility and precision (14). SILAC pairs are identical and only differ in their mass; consequently, they co-elute in high performance liquid chromatography but are distinguished using a mass spectrometer. The relative quantification of the peptides results from comparisons between the intensities of the neighbouring SILAC pairs, and the identification results from the fragmentation of either the light or heavy version of the same SILAC peptide (13).

In the present study, incipient CRC metastatic cells were compared with non-metastatic CRC cells using the SILAC method and an MS-based analysis in order to identify and quantify the early proteomic changes that occur in the metastatic cells. Among all of the proteins that were detected as deregulated in metastatic cancer cells with respect to primary cancer cells, those belonging to nicotinamide adenine dinucleotide hydride (NADH) dehydrogenase complex I were studied in detail. A major energy requirement for metastatic cells was associated with the overexpression of NADH dehydrogenase complex I proteins, as postulated by the Warburg effect (15). However, during the analysis of functionality, it was observed that it was a vestige of the cell transformation that was not functional at all.

\section{Materials and methods}

Animals. A total of 24 syngeneic BALB/c mice (male; 6-8 weeks old; weight, 25 g) were obtained from Charles River (Barcelona, Spain). Animal housing, care and experimental conditions were conducted in conformity with institutional guidelines and international laws following the criteria outlined in the 'Guide for the Care and Use of Laboratory Animals' prepared by the National Academy of Sciences and published by the National Institutes of Health (16). The animals were fed with standard chow and water ad libitum and were housed at $20-21^{\circ} \mathrm{C}$ and $55-65 \%$ humidity with a 12-h light/dark cycles. The animal experiments performed in the present study were approved by the Ethical Committee for Animal Experiments of the University of the Basque Country (Leioa, Spain; no. CEBA/237/2012/BADIOLA ETXABURU).

Cell culture. Murine colon carcinoma C26.WT cells (American Type Culture Collection, Manassas, VA, USA) were maintained at $37^{\circ} \mathrm{C}$ and $5 \% \mathrm{CO}_{2}$ in RPMI-1640 medium (Sigma-Aldrich; Merck KGaA, Darmstadt, Germany) supplemented with $10 \%$ foetal bovine serum (FBS; Gibco; Thermo Fisher Scientific Inc., MA, USA), and $1 \%$ penicillin/streptomycin (p/s; Sigma-Aldrich; Merck KGaA).

Experimental liver metastasis. Hepatic metastases were produced by intrasplenic inoculation of $1.5 \times 10^{6}$ colorectal carcinoma C26.WT cells into anaesthetized syngeneic $\mathrm{BALB} / \mathrm{c}$ mice, as previously reported (17). Mice were sacrificed 15 days following tumour development and two different types of tumours (the spleen primary tumour and liver metastatic tumour) were carefully dissected and extracted from each organ (Fig. 1). The dissected tumours were cultured on a petri dishes with $0.25 \%$ trypsin-EDTA for $5 \mathrm{~min}$ at $37^{\circ} \mathrm{C}$ and $5 \% \mathrm{CO}_{2}$, and following extensive washing with PBS, disaggregated viable cells were maintained in RPMI-1640 medium supplemented with $10 \%$ FBS and gentamicin $(10 \mu \mathrm{l} / \mathrm{ml}$; Sigma-Aldrich; Merck KGaA). 
SILAC labelling and protein extraction. For SILAC experiments, the RPMI media was custom-made and deficient for L-Arg and L-Lys (Thermo Fisher Scientific, Inc., Waltham, MA, USA). The media was supplemented with $10 \%$ dialyzed serum (Thermo Fisher Scientific, Inc.), 1\% p/s (Merck KGaA), $10 \mu \mathrm{l} / \mathrm{ml}$ gentamicin (Merck KGaA) and different isotopes of lysine and arginine (Lys0/Arg0 or Lys4/Arg6).

Cells were lysed with ice-cold co-immunoprecipitation buffer (25 mM Tris-HCl pH 7.5, $100 \mathrm{mM} \mathrm{NaCl}, 1 \% \mathrm{NP}-40,1 \mathrm{mM}$ sodium pervanadate, $5 \mathrm{mM} \beta$-glycerophosphate and $5 \mathrm{mM}$ $\mathrm{NaF}$ ) and complete protease inhibitor cocktail (complete tablets; Roche Diagnostics, Basel, Switzerland), and protein concentrations were estimated using a BCA protein assay (Thermo Fisher Scientific, Inc.). Protein lysates corresponding to differentially labelled primary and metastatic C26.WT cells were combined in a protein concentration ratio of 1:1 (primary Lys $0 / \mathrm{Arg} 0$ :metastatic Lys4/Arg6intwoof thereplicates and primary Lys4/Arg6:metastatic Lys0/Arg0 in one additional replicate).

SDS-PAGE and in-gel digestion. Protein lysates were run in two parallel lanes of a precast NuPAGE $4-12 \%$ Bis-Tris gel (Thermo Fisher Scientific, Inc.) and visualized with colloidal blue (Thermo Fisher Scientific, Inc.). Both gel lanes were separately cut into slices and subjected to in-gel reduction, alkylation and $5 \mathrm{ng} / \mu \mathrm{l}$ trypsin digestion as previously described (18). Derived peptides were concentrated and desalted using C18 STAGE Tips and further analysed by liquid chromatography (LC)-MS/MS. Experiments were performed in duplicate, with the biological conditions reversed between light and heavy SILAC labels.

In-solution digestion and pISep fractionation. Protein lysates derived from primary (Lys0/Arg0) and metastatic (Lys4/Arg6) cancer cells were combined and digested in solution using $5 \mathrm{ng} / \mu \mathrm{l}$ LysC and $5 \mathrm{ng} / \mu \mathrm{l}$ trypsin, as previously described (19). Tryptic peptides were then acidified with trifluoroacetic acid (TFA) to a final concentration of $0.3 \%$ and concentrated using a $\mathrm{C}_{18}$ Sep-Pak cartridge (Waters Corporation, Milford, MA, USA), according to the manufacturer's instructions. The organic solvent was evaporated using SpeedVAC, and then peptides were subjected to $\mathrm{pH}$ gradient fractionation (pISep; CryoBioPhysica, Rockville, MD, USA). Briefly, the peptide mixture was diluted twice with buffer A [pISep concentrate A (CryoBioPhysica), 25\% acetonitrile (ACN), $1 \%$ acetic acid, $\mathrm{pH} 2.8$ ] and loaded onto a small column containing a mixture of strong-cation and weak-cation exchange material (PolySULFOETHYL A ${ }^{\mathrm{TM}}$ and PolyCAT A ${ }^{\mathrm{TM}}$; PolyLC Inc., Columbia, MD, USA). Peptides were eluted by a stepwise increase in the $\mathrm{pH}$ of the elution buffer from $\mathrm{pH} 3.5$ to 10.0 that was achieved by mixing buffers A and B [pISep concentrate $\mathrm{B}$ (CryoBioPhysica), $25 \%$ acetonitrile, $0.5 \%$ ammonium hydrochloride, $\mathrm{pH}$ 10.8] in different volumes. The flow-through and 11 elution fractions were collected for each technical replicate. Each fraction was dried down in a SpeedVAC and adjusted to a final concentration of $1.7 \%$ ACN $/ 0.33 \%$ TFA. Then, the peptides were concentrated and desalted using $\mathrm{C}_{18}$ STAGE tips and further analysed by LC-MS/MS.

LC-MS/MS analysis. LC-MS/MS analysis of peptides that had previously been concentrated and desalted was performed using a reversed-phase liquid chromatography system (EASY-nLC 1000 ultra-high pressure; Thermo Fisher Scientific, Inc.) interfaced with a Q Exactive mass spectrometer (Thermo Fischer Scientific, Inc.) via a nanoelectrospray source (Thermo Fisher Scientific, Inc.). Acidified peptides were loaded on an analytical in-house packed column (20 cm x75 $\mu \mathrm{m}$; ReproSil-Pur $\mathrm{C}_{18}$-AQ $3 \mu \mathrm{m}$ resin; Dr. Maisch HPLC GmbH, Ammerbuch, Germany) in solvent $\mathrm{A}(0.5 \%$ acetic acid) and eluted by a nonlinear 120 min solvent B gradient $(0.5 \%$ acetic acid, $80 \%$ $\mathrm{ACN}$ ) at a flow rate of $250 \mathrm{nl} / \mathrm{min}$. Q Exactive was operated in a top 10 data-dependent mode. Survey scans were acquired at a resolution of $70,000(\mathrm{~m} / \mathrm{z} 400)$ and fragmentation spectra at $35,000(\mathrm{~m} / \mathrm{z} 400)$. MS/MS data were acquired in negative ion mode. Precursors were fragmented by higher energy C-trap dissociation (HCD) with a normalized collision energy of $25 \mathrm{eV}$. The maximum injection time was $120 \mathrm{msec}$ for the survey and $124 \mathrm{msec}$ for the MS/MS scan, where the AGC target values of 1e6 and 1e4 were used for survey scans and for MS/MS scans, respectively. In the Velos Orbitrap MS system survey, full-scan MS spectra (m/z range, 300-1,750; resolution 30,000 at m/z 400) were acquired and the 8 most intense multiple charged ions were fragmented by HCD (resolution 15,000 at m/z 400). Repeat sequencing of the peptide was minimized by excluding the selected peptide candidates for $45 \mathrm{sec}$. All raw data files acquired were searched against the UniProt (www.uniprot. org/) mouse database version 2014.01 (with 88,479 sequence entries) with a MaxQuant proteomics computational platform version 1.3.0.5 and using the Andromeda search engine (Cox and Mann ref). In the SILAC experiments, light and heavy labels were set as $\operatorname{Arg} 0 / \mathrm{Lys} 0$ and $\operatorname{Arg} 6 / \mathrm{Lys} 4$. Precursor and fragment mass tolerances were set to 7 and $20 \mathrm{ppm}$, respectively. Enzyme specificity was set to trypsin, allowing for cleavage of the $\mathrm{N}$-terminal to proline and between aspartic acid and proline (with a maximum of 2 missed cleavages). Carbamidomethylation of $\mathrm{C}$ was set as a fixed modification, whereas oxidation of $\mathrm{M}$, protein $\mathrm{N}$-terminal acetylation and NQ deamidation were selected as variable modifications for database searching. For peptide and protein identification a minimal peptide length of 7 amino acids was required, and the false discovery rate was set at 0.01 . Additionally, for protein identification at least two peptides were required, of which at least one was unique to the protein group. The razor and unique peptides were considered for protein quantification.

Migration assay. Metastatic and primary C26.WT cells were seeded on three collagen (Sigma-Aldrich; Merck KGaA) precoated transwell cell culture inserts (Greiner Bio-One North America Inc., Monroe, NC, USA) in 24-well culture plates with RPMI-1640 without serum in the upper chamber at a concentration of $2 \times 10^{5}$ cells $/ \mathrm{ml}$, and RPMI-1640 with $0.5 \%$ FBS (Gibco; Thermo Fisher Scientific Inc.) was plated in the lower chamber. Following $18 \mathrm{~h}$ of incubation, the migration levels were assessed by staining the chambers with eosin at room temperature for $10 \mathrm{~min}$. The number of migrated cells was estimated under a light microscope (magnification, $\mathrm{x} 20$ ) using ImageJ 1.48v software (National Institutes of Health, Bethesda, MD, USA). The experiment was repeated three times.

Western blotting. Western blot analysis was performed with cell lysates from primary and metastatic tumour cells. Cell 
lysates were obtained with Radioimmunoprecipitation Assay lysis buffer (Sigma-Aldrich; Merck KGaA) and the protein concentration was determined by a Pierce BCA Protein Assay kit (Thermo Fisher Scientific, Inc.). A total of $25 \mu \mathrm{g}$ of each cell lysate was separated by $10 \%$ SDS-PAGE, and blotted onto a polyvinylidene difluoride (PVDF) membrane (Bio-Rad Laboratories, Hercules, CA, USA). Then, the membrane was blocked for $1 \mathrm{~h}$ at room temperature with TBS-T (Tween $0.5 \%$ ) in $5 \%$ milk and incubated overnight at $4{ }^{\circ} \mathrm{C}$ with the following primary antibodies: H3K4me3 (1:300; Abcam, Cambridge, MA, USA; cat. no. ab12209), H3K9me3 (1:1,000; Abcam; cat. no. ab8898) and histone 3 lysine 27 trimethylated (H3K27me3; 1:500; Abcam; cat. no. ab6002). Histone 3 (1:1,000; Sigma-Aldrich; Merck KGaA; cat. no. H0164) was used as the control. Following extensive washing with $1 \%$ TBS-T, membranes were incubated for $1 \mathrm{~h}$ at room temperature with the corresponding secondary antibody: Horseradish peroxidase-conjugated goat anti-mouse (1:1,000; Sigma-Aldrich Merck KGaA; cat. no. AP308P) or goat anti-rabbit (1:3,000; Abcam; cat. no. ab6721). The membranes were developed with an enhanced chemiluminescence (ECL) system (GE Healthcare, Chicago, IL, USA), and the images were analysed using the transluminator Gel Doc 2000 (Bio-Rad Laboratories) and Gel Documentation System (with Image Lab $^{\mathrm{TM}}$ software 5.0; Bio-Rad Laboratories).

NADH assay. The NADH colorimetric assay was performed according to the manufacturer's instructions (ScienCell Research Laboratories, Inc., San Diego, CA, USA; cat. no. 8368). Briefly, a standard curve was prepared using the provided material. Independently, the working reagent was prepared by mixing $76 \mu \mathrm{l}$ assay buffer, $10 \mu \mathrm{l}$ lactate solution, $2 \mu 1$ 2-(4-iodophenyl)-3-(4-nitrophenyl)-5-phenyl-2H-tetrazolium and $2 \mu \mathrm{l}$ enzyme mix. Next, $90 \mu \mathrm{l}$ of the working reagent was mixed into each well of the 96-well plate containing the NAD standard, test samples and blank. Plates were incubated for $20 \mathrm{~min}$ at room temperature and were protected from light; the absorbance was read at a wavelength of $490 \mathrm{~nm}$ on an ELISA plate reader.

Intracellular reactive oxygen species (ROS) measurement. C26.WT cells were cultured in a 24-well plate at a concentration of 100,000 cells/well. Then, the accumulation of ROS within cells was measured by labelling cells for $1 \mathrm{~h}$ at $37^{\circ} \mathrm{C}$ and $5 \% \mathrm{CO}_{2}$ with $10 \mu \mathrm{M}$ 5,6-chloromethyl-20,70-dichlorodihydrofluoresceindiacetate (CM-DCFDA; Molecular Probes; Thermo Fisher Scientific, Inc.; cat. no. C6827). The total cell number was determined by staining cells with $5 \mu \mathrm{l} / \mathrm{ml}$ Hoechst (Molecular Probes; Thermo Fisher Scientific, Inc.; cat. no. $\mathrm{H} 1398$ ) for $1 \mathrm{~h}$ at $37^{\circ} \mathrm{C}$ and $5 \% \mathrm{CO}_{2}$. Finally, fluorescence was measured using a Fluoroskan Ascent plate fluorimeter (Thermo Labsystems, Santa Rosa, CA, USA), and data are expressed as a normalized percentage of CM-DCFDA/Hoechst fluorescence.

Mitochondrial potential measurement. Primary and metastatic tumour cells were cultured in 24-well plates, loaded with $100 \mathrm{nM}$ tetramethylrhodamine ethyl ester (TMRE; Molecular Probes; Thermo Fisher Scientific, Inc.; cat. no. T669) and maintained for $1 \mathrm{~h}$ at $37^{\circ} \mathrm{C}$ and $5 \% \mathrm{CO}_{2}$. TMRE is a highly membrane-permeant cationic fluorophore that accumulates in negatively charged subcellular compartments, notably mitochondria. Under non-quenching conditions, quantifying the retention of the dye by whole cells provides an estimate of their average mitochondrial potential. As aforementioned, Hoechst fluorescence was used to quantify the number of cells present within the reading field. Fluorescence was measured using a Fluoroskan Ascent plate fluorimeter (Thermo Labsystems), and the data were expressed as a normalized percentage of TMRE/Hoechst fluorescence.

Statistical analysis. Statistical analysis was performed with Student's two-tailed unpaired t-tests using Excel 2016 (Microsoft Corporation, Redmond,WA, USA). All experiments were carried out in triplicate, and data were expressed as the mean \pm standard deviation. $\mathrm{P}<0.05$ was considered to indicate a statistically significant difference.

\section{Results}

C26.WT murine colon cancer cells extracted from the liver exhibit metastatic characteristics. Metastatic and non-metastatic colon cancer cells were obtained using a murine liver metastasis model. The in vivo experiments were performed in duplicate with 6 animals/group (total $n=24$ animals). Animals were injected with the C26.WT murine colon cancer cell line intrasplenically. Following 15 days, the cancer cells remaining in the spleen, as well as those in the liver, were collected (Fig. 1). All of the animals injected with cancer cells directly into the spleen following inoculation exhibited primary tumours in the spleen and metastatic liver tumours.

A general characteristic of metastatic cells is their capacity to migrate (20). To confirm that the cancer cells extracted from the liver were metastatic, the present study measured their capacity to migrate. Primary as well as metastatic cancer cells extracted from the spleen and liver, respectively, were incubated on a collagen type I-covered $8 \mu$ m-diameter pore membrane. Following $18 \mathrm{~h}$, the number of cells that had crossed over the pores were quantified. It was revealed that liver tumour cells migrated 4 times more than primary tumour cells, which was in agreement with their increased invasive capacity (Fig. 2).

Additionally, epigenetic changes are widely associated with cancer (21) and specifically the demethylation of histone 3 at lysine 27 has been described to influence colorectal liver metastatic capacity (22). Therefore, to validate the metastatic capacity of cancer cells, the present study detected and compared the presence of $\mathrm{H} 3 \mathrm{~K} 27 \mathrm{me} 3$ in colorectal cancer cells isolated from the spleen, and colorectal cancer cell growth in the liver by western blotting. As shown in Fig. 2B, the cancer cells isolated from the liver had reduced levels of H3K27me3 when in comparison with those of cells extracted from the spleen. These results indicated that the cancer cells growing in the liver were metastatic cancer cells.

Differential proteomics of metastatic and non-metastatic colon cancer cells. To understand the molecular events that trigger the metastatic capacity of the colon cancer cells observed in the liver, the present study performed SILAC-based quantitative proteomics analysis and compared the proteome 


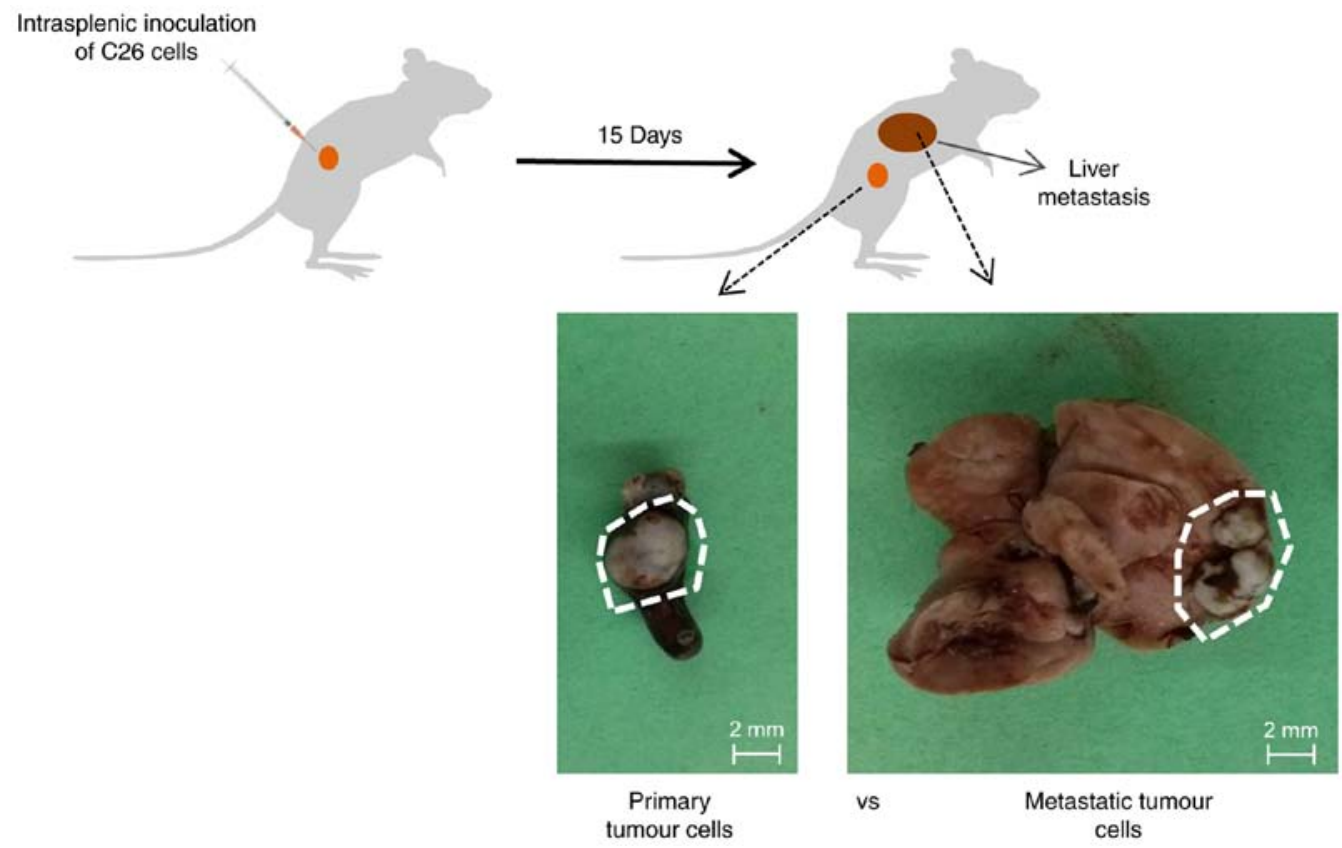

Figure 1. Schematic representation of the metastasis model modified from the model of Fidler (3).

A
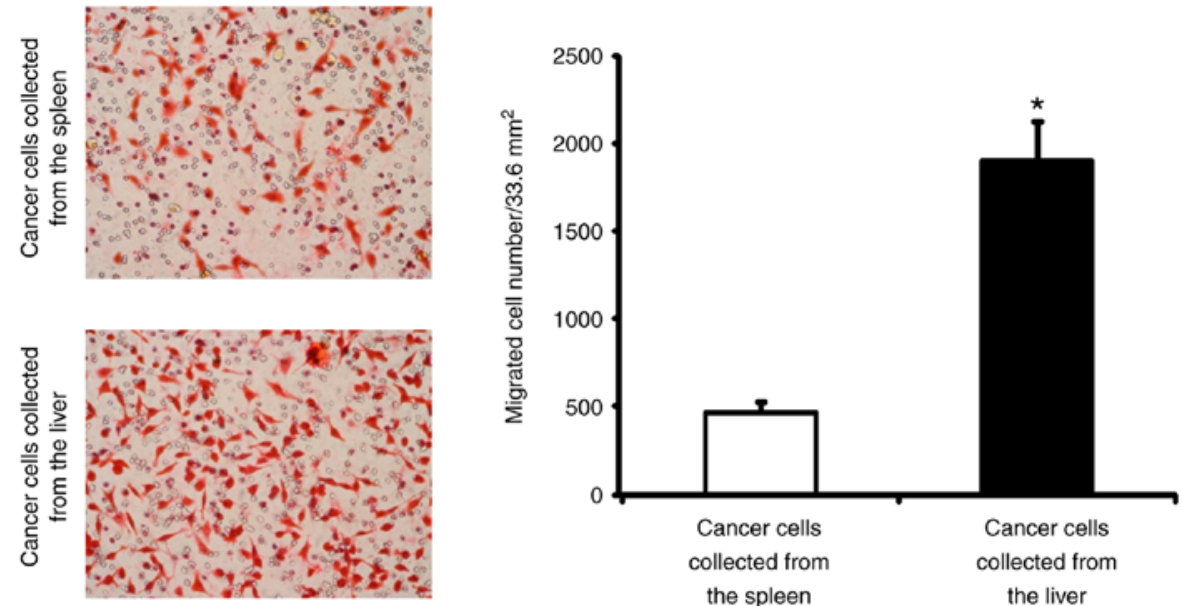

B

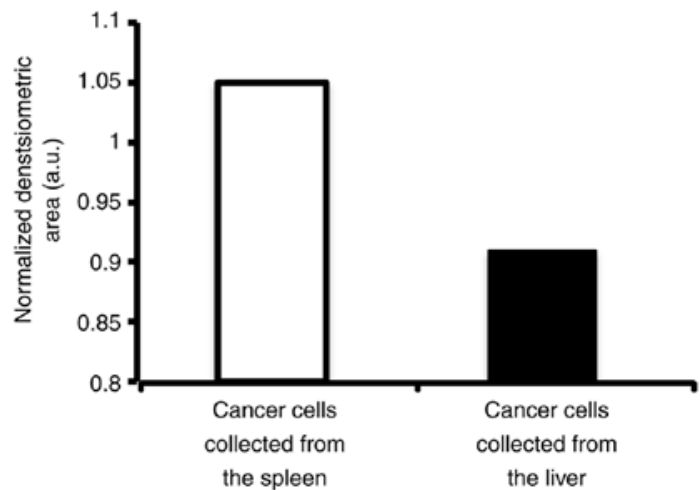

Figure 2. Characterization of cells collected in the spleen and the liver. (A) Migration assay of C26.WT cells collected in the liver and the spleen following liver metastatic model experiments (magnification, $\mathrm{x} 10$ ). " $\mathrm{P}<0.05$ vs. cancer cells isolated from the spleen. (B) Western blot analysis was performed to detect H3K27 trimethylation of cells collected in the spleen and the liver. Histone 3 expression was used as a control to normalize the expression in the representative diagram. H3K27me3, histone 3 lysine 27 trimethylated; H3, histone 3.

of metastatic and non-metastatic colon cancer cells extracted from the spleen and liver. Briefly, extracted primary and metastatic cancer cells were grown in the presence of either light or heavy Lys and Arg amino acids. Once their proteomes 
A

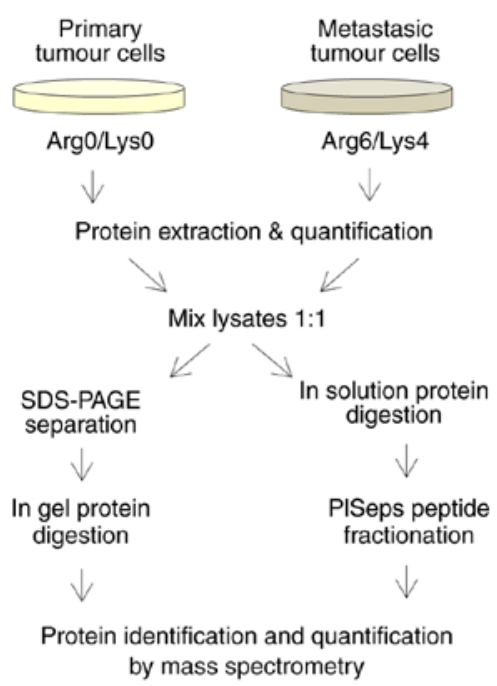

B
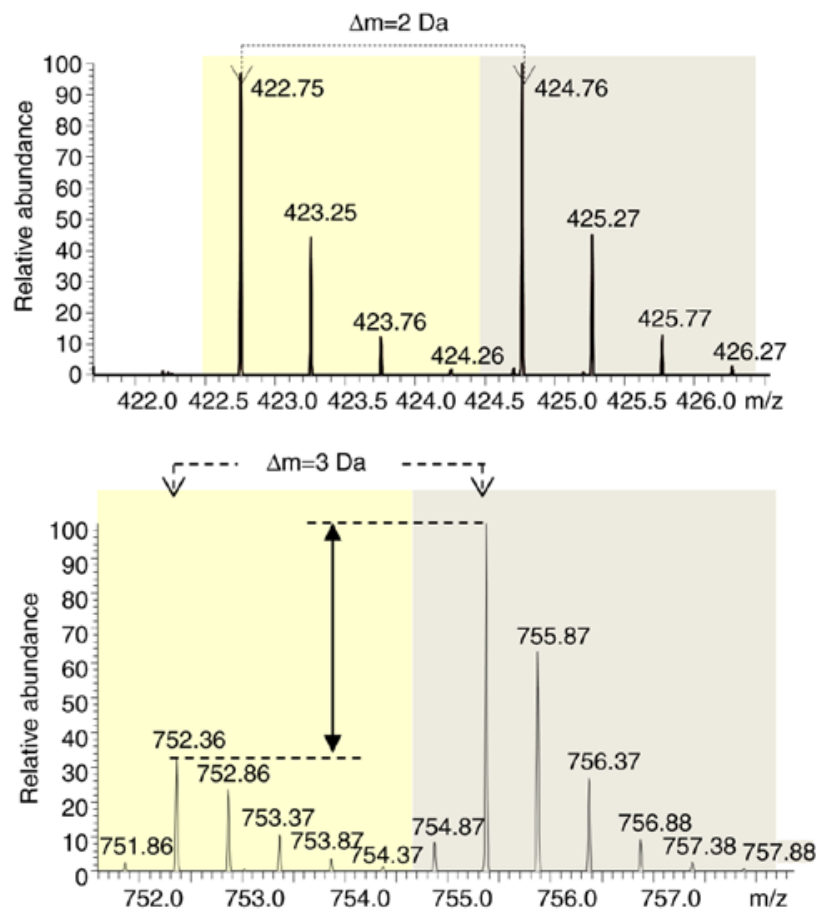

Figure 3. Schematic diagram of the experimental procedure and representative mass spectra. (A) Flow diagram of the MS-based quantitative proteomics strategy to compare the proteome of primary and metastatic cancer cells. (B) Examples of stable isotope labelling of amino acids in cell culture MS spectra illustrating peptides that display different ratios. (Top-panel) The Lys-containing peptide ( $\mathrm{z}=+2)$ presenting a 1:1 ratio; (bottom-panel) the Arg-containing peptide $(\mathrm{z}=+2)$ was upregulated in metastatic cells. MS, mass spectrometry.

A

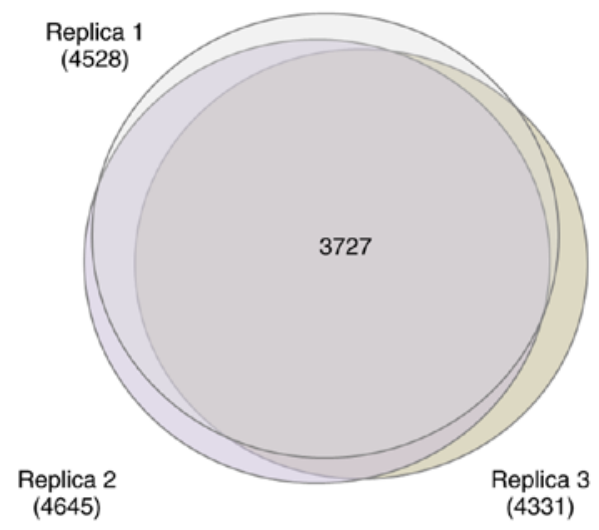

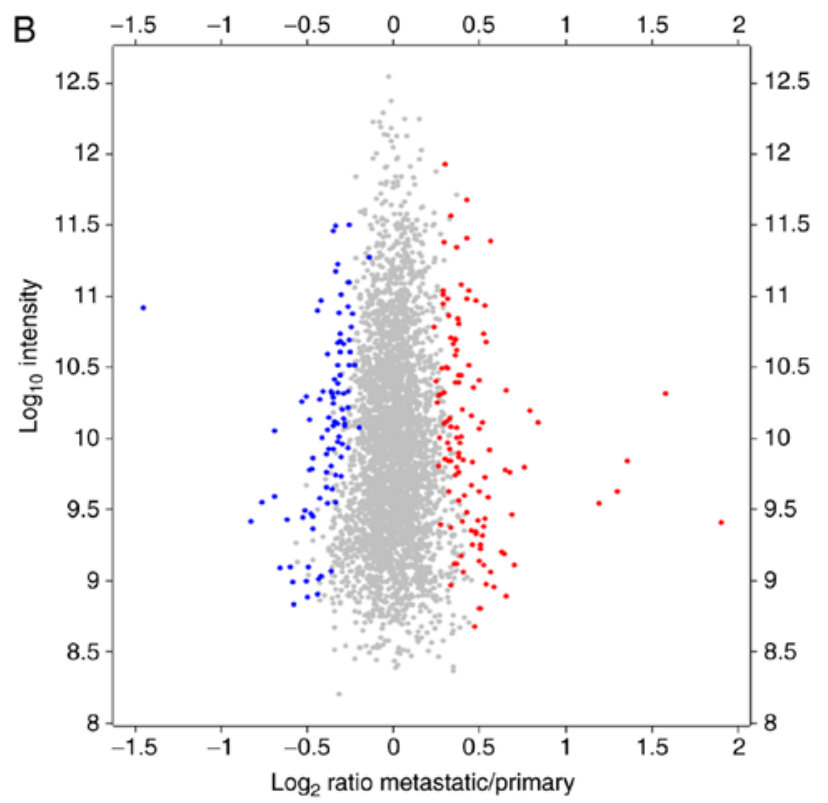

Figure 4. Mass spectrometry-based differential proteomic analysis of primary and metastatic cancer cells. (A) Venn diagram, demonstrating overlap in proteins quantified in the three SILAC replicates. (B) Overall metastatic/primary SILAC ratio as a function of protein intensity. Indicated in blue and red are the proteins significantly $(\mathrm{P}<0.05)$ down- and upregulated in metastatic cancer cells, respectively, in comparison to non-metastatic cancer cells. SILAC, stable isotope labelling of amino acids in cell culture.

were labelled, proteins were extracted, quantified and equivalently combined. Half of the mixed sample was fractionated by SDS-PAGE, the proteins were in-gel digested, and the resultant peptides were analysed by LC-MS/MS. The other half of the mixed sample was in-solution digested, and the resultant peptides were fractionated using the PISep procedure prior to MS analysis (Fig. 3A).
The present study performed three independent SILAC experiments following two complementary strategies, resulting in the identification and quantification of 5,257 proteins in total (Fig. 3B). The identification was based on strict criteria, using a false discovery rate of 0.01 for peptides and proteins, and at least 2 peptides including 1 unique peptide for positive identification, whereas razor and unique peptides 

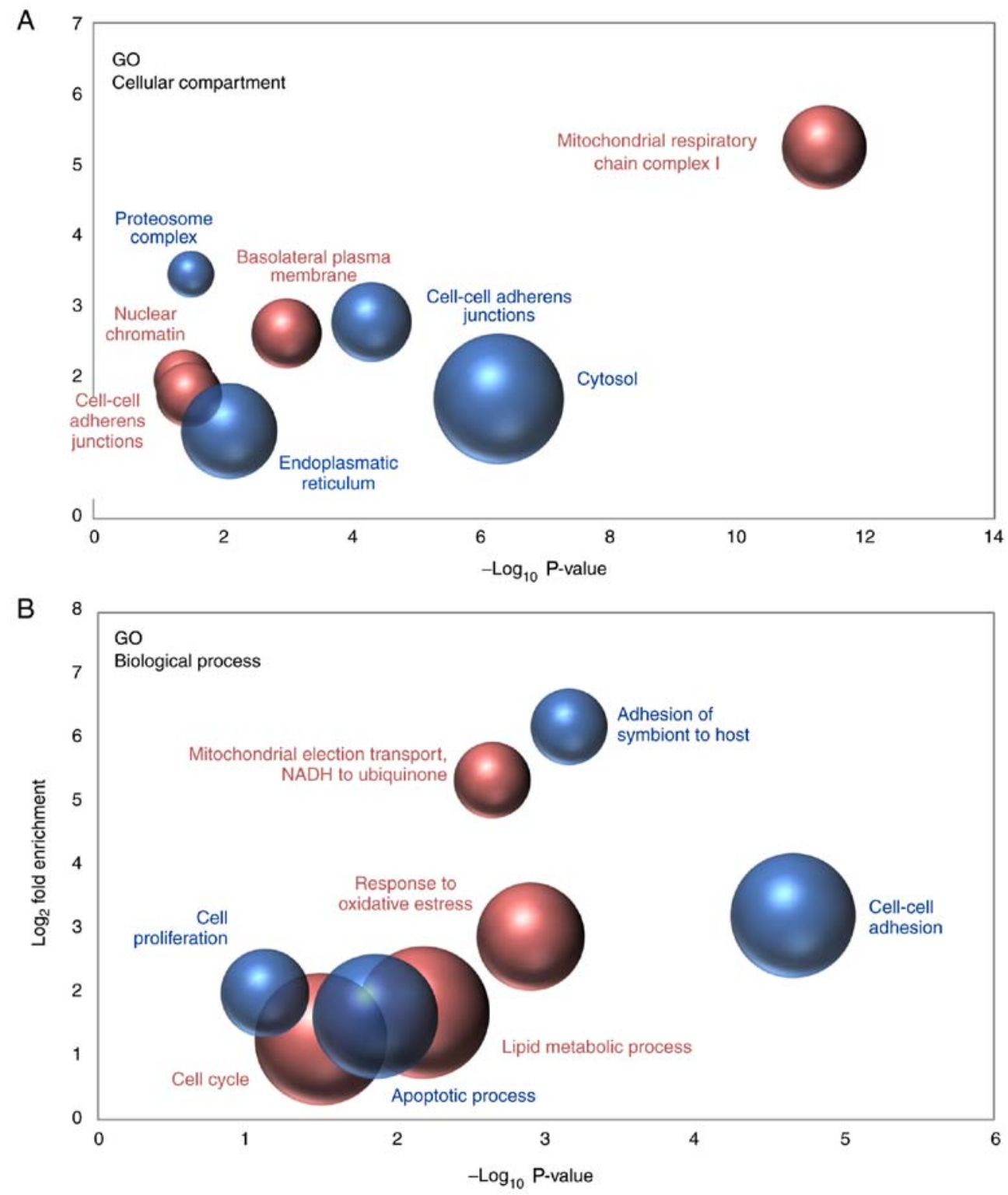

Figure 5. GO analysis indicating the (A) 'Cellular compartments' and (B) 'Biological processes' overrepresented within the proteins significantly ( $\mathrm{P}<0.05)$ upregulated (in red) and downregulated (in blue) in metastatic cancer cells with respect to non-metastatic cancer cells. The fold enrichment and the statistical significance of the most indicative terms are shown. The size of the dots correlates with the number of proteins grouped in the same term. GO, Gene Ontology.

were subjected to quantitation. From these 5,257 proteins, 3,727 were commonly quantified in the three replicates performed (Fig. 4A).

To detect the proteins that were differentially expressed in primary and metastatic cancer cells, the significance of each protein quantified in each independent replicate was calculated using Perseus from MaxQuant software. The present study focused on the proteins that were significantly $(\mathrm{P}<0.05)$ changed in abundance between primary and metastatic cells in at least 2 out of the 3 replicates performed. In total, 120 and 95 proteins were observed to be consistently upregulated and downregulated, respectively, in metastatic cancer cells with respect to primary cancer cells (Fig. 4B).

The proteins that were detected to be differentially expressed in metastatic and primary cancer cells were functionally classified by Gene Ontology (GO) analysis, with the aim to detect the most significantly enriched terms within the 'Cellular Compartment' and 'Biological Process' categories. Regarding the 'Cellular Compartment' category, the results indicated that the nature of the proteins observed to be upregulated and downregulated in metastatic cells when compared with non-metastatic cancer cells was quite different. Downregulated proteins were primarily proteins that were localized in cell-to-cell adherens junctions, the cytosol, the endoplasmic reticulum and the proteasomal complex; however, proteins that were upregulated in metastatic cancer cells mainly belonged to mitochondrial respiratory chain complex I and, to a lesser extent, to nuclear chromatin and the basolateral plasma membrane (Fig. 5A). In line with these results, various biological processes associated with cell adhesion were significantly overrepresented among the downregulated proteins. By contrast, the analysis demonstrated that the mitochondrial electron transport from NADH to ubiquinone was the most enriched biological process among the proteins observed to be upregulated in metastatic cancer cells (Fig. 5B). 


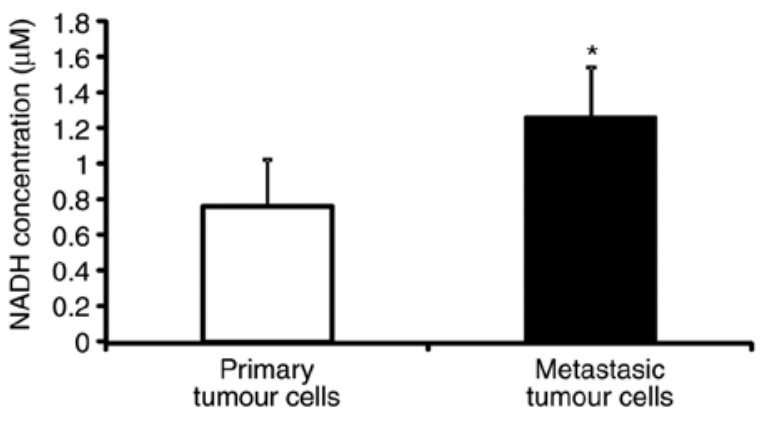

Figure 6. NADH concentration measurement in primary cells with respect to metastatic cells. ${ }^{*} \mathrm{P}<0.05$ vs. primary tumour cells. NADH, nicotinamide adenine dinucleotide hydride.

NADH concentration is higher in metastatic C26.WT tumour cells than in non-metastatic cells. The SILAC-based differential proteomics study presented above uncovered that the NADH dehydrogenase complex, or respiratory complex I, which is a key component of the cell, is expressed to a greater extent in metastatic cells than in non-metastatic C26.WT cancer cells. Thus, the present study then set out to verify if the difference in quantity that was detected was associated with a functional difference in the activity of the complex. NAD (the oxidized and reduced forms abbreviated as $\mathrm{NAD}^{+}$and $\mathrm{NADH}$, respectively) is a key enzymatic cofactor involved in a number of redox reactions that acts as a soluble electron carrier. As NADH deposits electrons at the respiratory complex $\mathrm{I}$, and thereby becoming $\mathrm{NAD}^{+}$, the present study measured the concentration of NADH in metastatic and non-metastatic cancer cells as an indicator of the activity of the NADH dehydrogenase complex. The NADH colorimetric assay performed revealed that the concentration of NADH was almost twice as high in metastatic cancer cells with respect to primary tumour cells, indicating a possible dysfunction of respiratory complex I between the two cancer cell types (Fig. 6).

Production of ROS is higher in metastatic than in non-metastatic C26.WT tumour cells. Mitochondrial NADH dehydrogenase complex dysfunction is associated with the production of ROS (23). ROS are known mutagens whose production accelerates cellular conversion to a metastatic phenotype (24-26). Since an increased NADH concentration in metastatic tumour cells was observed, it was postulated whether the higher rate of ROS generation was due to a dysfunction in mitochondrial NADH dehydrogenase activity. To investigate this idea, ROS levels were quantified in primary and metastatic tumour cells. Briefly, C26.WT cells were cultured with the CM-DCFDA probe. This probe is non-fluorescent, but in the presence of ROS following oxidation, it becomes fluorescent inside ROS-accumulating cells. As shown in Fig. 7A, a significant increase in CM-DCFDA fluorescence in $\sim 25 \%$ of liver metastatic cells was observed, which confirmed that basal ROS generation was enhanced in these metastatic cells with respect to primary tumour cells.

Mitochondrial membrane potential is lower in C26.WT metastatic tumour cells than in non-metastatic C26.WT
A
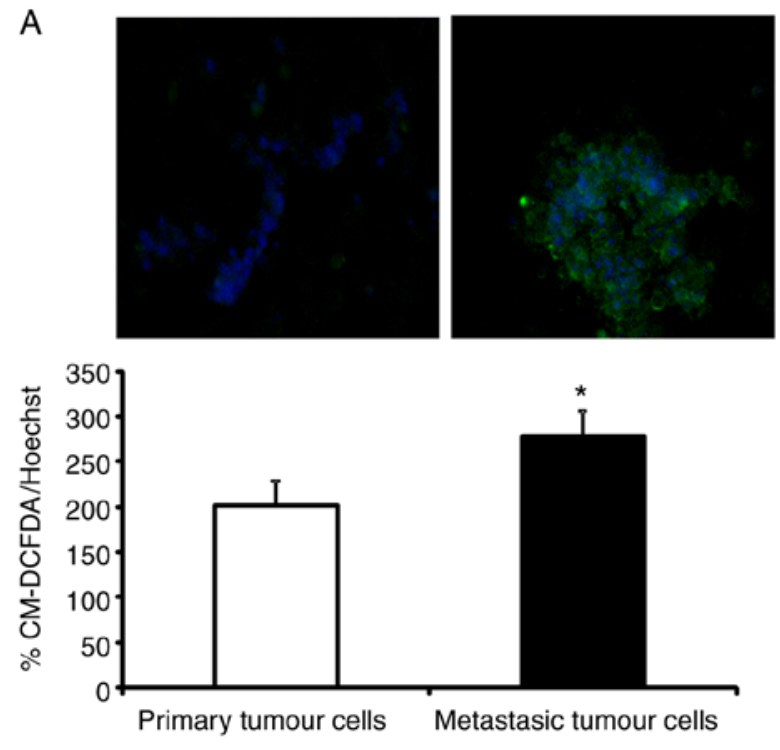

B
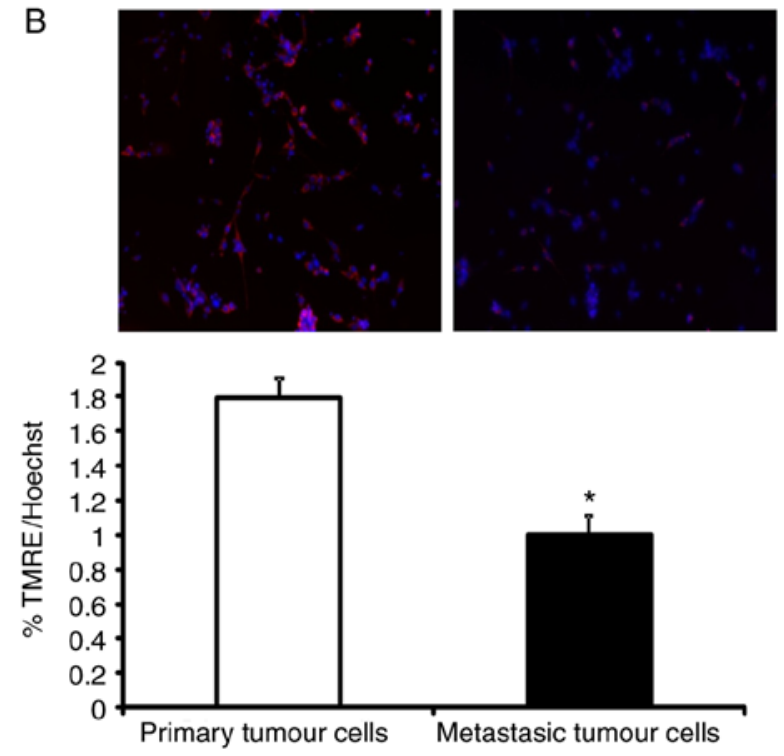

Figure 7. Warburg effect characterization in metastatic cells with respect to primary tumour cells. (A) ROS measurement in C26.WT primary cells with respect to metastatic cells. Green staining indicates a higher ROS concentration; blue staining indicates nuclei (DAPI; magnification, x20). (B) Mitochondrial membrane potential measurement in metastatic cells with respect to primary cells. Violet staining indicates more mitochondrial membrane potential and greater mitochondrion integrity; blue staining indicates nuclei (DAPI; magnification, $\mathrm{x} 20$ ). ${ }^{*} \mathrm{P}<0.05$ vs. primary tumour cells. ROS, reactive oxygen species; CM-DCFDA, 5,6-chloromethyl-20,70-dichlorodihydrofluoresceindiacetate; TMRE, tetramethylrhodamine ethyl ester.

tumour cells. The generation of ROS is closely associated with alterations in the mitochondria. Due to an increase in ROS generation, NADH accumulation and the increased expression of NADH dehydrogenase complex subunits in metastatic tumour cells, the functionality of mitochondria in these cells was analysed. Damaged or low-efficiency mitochondria decrease their oxidative phosphorylation rate and instead generate more ROS (27). To test this hypothesis, the mitochondrial membrane potential was measured using the fluorescent probe TMRE. TMRE is a cell-permeant, positively charged probe that accumulates in active mitochondria due to their relatively negative charge. When mitochondria 
are depolarized or inactive, they decrease their membrane potential and fail to sequester TMRE (28). As shown in Fig. 7B, metastatic tumour cells decreased by $45 \%$ of the uptake of TMRE when in comparison with primary tumour cells. This confirmed that mitochondria in metastatic tumour cells were more depolarized (and thus, less efficient) than those of primary tumour cells, despite their increased expression of mitochondrial NADH dehydrogenase complex I subunits.

\section{Discussion}

In the present study, a murine experimental CRC liver metastasis model was generated to study the molecular mechanism implicated in cell transformation. The aim was to investigate the early stages of the metastatic transformation in order to better understand the transformation process.

First, the metastatic phenotype of tumour cells was verified by analysing the invasiveness of the cells. It is known that one of the most important characteristics of cancer metastatic cells is motility (29). Metastatic cells have the capacity to move from the primary organ where they are growing as a primary tumour, to invade the blood vessels and then to colonize in distant organs as metastatic tumour cells. In accordance with this, the liver metastatic C26.WT cells exhibited a higher motility than the primary C26.WT tumour cells. Furthermore, the characterization of the two populations (primary and metastatic tumour cells) was verified by analysing their histone methylation profiles. Histone methylation is an epigenetic mechanism that reprogrammes the cells and has been implicated in tumourigenesis and metastasis (21). Different histone modifications alter the charge distribution of these proteins, leading to spatial changes with consequent rearrangement of the chromatin. This chromatin reorganization influences gene expression, either allowing or repressing the expression of different genes (21). It has been previously observed that the malignancy of certain cancer cells is a consequence of chromatin remodelling, with the subsequent activation or inactivation of oncogenes and tumour suppressor genes, respectively (30). Histone 3 has been well studied within this field, and more specifically, lysine 27 of this protein has also been investigated extensively. Low trimethylation status of the aforementioned amino acid of histone 3 is associated with poor prognosis and metastatic phenotype In many types of cancer $(22,31,32)$. In addition, the enhancer of zeste 2 , a methyltransferase and a component of the polycomb repressive complex 2 , serves an essential role in the epigenetic maintenance of H3K277me3. This modification produces chromatin condensation and converts the involved DNA-region into heterochromatin, thereby decreasing gene transcription (33). As described previously, the metastatic potential of many types of cancer is associated with the low expression of H3K27 me3, which is due to the expression of prometastatic genes, that are normally repressed when lysine 27 of histone 3 is trimethylated. Liver metastatic colon cancer cells have decreased trimethylation of lysine 27 of histone 3 with respect to primary tumour cells purified from the spleen (32). This is in agreement with previous cases of pancreatic, ovarian and breast cancer, in which decreased trimethylation of lysine 27 of histone 3 was associated with higher invasiveness and more metastatic characteristics $(22,31,32)$.
Oxidative phosphorylation (OXPHOS) chain NADH dehydrogenase complex I overexpression is associated with a higher energy requirement and a more active, transforming cell phenotype (25). Thus, the SILAC analysis results support this hypothesis; however, when the functionality of the complex was analysed, non-functional complex I was detected by NAD accumulation and decreased TMRE values in metastatic cells. This loss of functionality led to the present study investigating the redox status in the two cell populations. According to Warburg et al (34), cancer cells have higher energy requirements than healthy cells; however, they utilize glycolysis instead of a more effective process such as oxidative phosphorylation. One explanation for the Warburg effect is that mitochondria accumulate defects during the process of cell transformation, rendering them less efficient. Thus, only cells that can switch to an enhanced glycolytic metabolism are able to survive within the tumour mass, which presents a compromised oxygen supply to the cells. This natural selection mechanism would favour the acquisition of more aggressive traits, such as an increased rate of glycolysis, decreased mitochondrial OXPHOS, increased production of ROS and an increased rate of DNA mutation. When the metabolisms of metastatic and primary tumour non-metastatic cancer cells were compared in the present study, the Warburg effect was aggravated in the metastatic cells. This was reflected by an increased accumulation of NADt, which was concurrent with a decrease in the mitochondrial membrane potential, as assessed by TMRE fluorescence. Cells that transform into more malignant and metastatic cells utilize glycolysis more and OXPHOS less in order to satisfy their energy demands, producing more ROS and reducing their mitochondrial membrane potential (15). This is notable as OXPHOS is a far more efficient energy generating mechanism than glycolysis. There are many hypotheses that explain why the Warburg effect benefits cancer cells. A previous study has suggested that cancer cells may inactivate their mitochondria in order to avoid apoptosis (35). Mitochondria are key organelles that serve an active role in some apoptotic pathways; thus, when they are inactivated tumour survival is facilitated. Another hypothesis was that tumour cells grow in anaerobic conditions, and therefore, they may evolve by switching their metabolism to anaerobic pathways. In addition, even when they grow in aerobic conditions this characteristic is irreversible (26). In the present study, an upregulation of OXPHOS complex I subunit expression in the metastatic phenotype cells was observed when compared with the non-metastatic phenotype cells. However, the results of the ROS generation and mitochondrial potential assays revealed that the mitochondrial respiratory chain in metastatic cells was dysfunctional; despite their elements being produced in higher amounts than in primary tumour cells, the mitochondrial potential was reduced, and ROS production was increased in these cells. As initially described by Warburg (34), the upregulation of anaerobic pathways would be more marked in cells that have a greater impairment of OXPHOS, which, while attempting to replace this lost energy source, evolve to become more aggressive and metastatic phenotypes.

The Warburg effect suggests that a primary tumour cell transforms into a metastatic tumour cell in two phases. In the first phase, primary tumour cells are not in completely 
anaerobic conditions, and thus, they are required to fulfil their energy requirement in order to grow. Therefore, they are forced to utilize the respiratory chain, upregulating the expression of the subunits of that chain, in order to acquire a more energetic phenotype. Then as the tumour mass reaches a critical level, the tumour microenvironment becomes anaerobic, and tumour cells enter the next phase. In this second phase, the cells are in an anaerobic microenvironment, and stop using the respiratory chain mechanism to produce energy and instead adopt anaerobic pathways as their primary energy sources. In this way, under hypoxic conditions tumour cells secrete hypoxic inducible factor-1 (HIF-1) and block the phosphatase and tensin homologue, activating the cell survival-associated protein kinase B signalling pathway (36). Furthermore, HIF-1 increases the gene expression of the glycolysis transporters glucose transporter 1 (GLUT1) and GLUT3, and blocks oxidative phosphorylation by inhibiting pyruvate entry into the tricarboxylic acid cycle (37). HIF-1 also regulates the tumour microenvironment via VEGF secretion, which induces endothelial cell transformation and angiogenic tumour support (37).

In conclusion, the upregulation of dysfunctional respiratory chain complex I may be a part of the preliminary phase of cell transformation, in which a high energy requirement and aerobic conditions were still present. Nevertheless, the characteristics observed in the metastatic C26.WT cell phenotype In the present study were in agreement with the Warburg effect, and this phenomenon could be explored in greater depth to develop novel therapeutic strategies. However, it should also be considered that these transforming cells upregulated ORPHOX elements during the first phase of malignancy.

\section{Acknowledgements}

The authors would like to thank the University of The Basque Country (Leioa, Spain) and University of Southern Denmark (Odense M, Denmark) for their support during the present study.

\section{Funding}

The present study was supported by grants from the Basque Government, Department of Health (grant no. 2011111023).

\section{Availability of data and materials}

The datasets used and/or analyzed during the current study are available from the corresponding author on reasonable request.

\section{Authors' contributions}

IB performed the cell biology and animal experiments, wrote the manuscript and managed the project. NO performed the SILAC experiments, wrote the manuscript and managed the project. JM conducted the cell biology and animal experiments, and contributed to writing the manuscript. VA performed the MS sample preparation experiments. IK contributed to the management of the project and performed the SILAC experiments. FU contributed to the project management, wrote the manuscript and performed the animal experiments. ASC conducted the cell biology and animal experiments, and wrote the manuscript. GI performed the cell biology experiments and wrote the manuscript. All authors read and approved the final manuscript and agree to be accountable for all aspects of the work in ensuring that questions related to the accuracy or integrity of any part of the work are appropriately investigated and resolved.

\section{Ethics approval and consent to participate}

The animal experiments in the present study were approved by the Ethical Committee for Animal Experiments of the University of Basque Country (no. CEBA/237/2012/BADIOLA ETXABURU).

\section{Patient consent for publication}

Not applicable.

\section{Competing interests}

The authors declare that they have no competing interests.

\section{References}

1. Trabulo D, Ribeiro S, Martins C, Teixeira C, Cardoso C, Mangualde J, Freire R, Gamito É, Alves AL, Augusto F, et al: Metabolic syndrome and colorectal neoplasms: An ominous association. World J Gastroenterol 21: 5320-5327, 2015.

2. Fancher TT, Palesty JA, Rashidi L and Dudrick SJ: Is gender related to the stage of colorectal cancer at initial presentation in young patients? J Surg Res 165: 15-18, 2011.

3. Fidler IJ: Selection of successive tumour lines for metastasis. Nat New Biol 242: 148-149, 1973

4. Gravekamp C, Sypniewska R, Gauntt S, Tarango M, Price P and Reddick R: Behavior of metastatic and nonmetastatic breast tumors in old mice. Exp Biol Med 229: 665-675, 2004.

5. Raz A, Hanna N and Fidler IJ: In vivo isolation of a metastatic tumor cell variant involving selective and nonadaptive processes. J Natl Cancer Inst 66: 183-189, 1981.

6. Rashidi B, Gamagami R, Sasson A, Sun FX, Geller J, Moossa AR and Hoffman RM: An orthotopic mouse model of remetastasis of human colon cancer liver metastasis. Clin Cancer Res 6: 2556-2561, 2000.

7. Vakiani E, Janakiraman M, Shen R, Sinha R, Zeng Z, Shia J, Cercek A, Kemeny N, D'Angelica M, Viale A, et al: Comparative genomic analysis of primary versus metastatic colorectal carcinomas. J Clin Oncol 30: 2956-2962, 2012.

8. Rapti SM, Kontos CK, Papadopoulos IN and Scorilas A: High miR-96 levels in colorectal adenocarcinoma predict poor prognosis, particularly in patients without distant metastasis at the time of initial diagnosis. Tumour Biol 37: 11815-11824, 2016.

9. Hinoue T, Weisenberger DJ, Lange CP, Shen H, Byun HM, Van Den Berg D, Malik S, Pan F, Noushmehr H, van Dijk CM, et al: Genome-scale analysis of aberrant DNA methylation in colorectal cancer. Genome Res 22: 271-282, 2012.

10. Tan HT, Wu W, Ng YZ, Zhang X, Yan B, Ong CW, Tan S, Salto-Tellez M, Hooi SC and Chung MC: Proteomic analysis of colorectal cancer metastasis: Stathmin-1 revealed as a player in cancer cell migration and prognostic marker. J Proteome Res 11: 1433-1445, 2012

11. Li G, Wang Z, Xu J, Wu H, Cai S and He Y: The prognostic value of lactate dehydrogenase levels in colorectal cancer: A meta-analysis. BMC Cancer 16: 249, 2016.

12. Tan GS, Lim KH, Tan HT, Khoo ML, Tan SH, Toh HC and Ching Ming Chung M: Novel proteomic biomarker panel for prediction of aggressive metastatic hepatocellular carcinoma relapse in surgically resectable patients. J Proteome Res 13: 4833-4846, 2014.

13. Ong SE, Blagoev B, Kratchmarova I, Kristensen DB, Steen H, Pandey A and Mann M: Stable isotope labeling by amino acids in cell culture, SILAC, as a simple and accurate approach to expression proteomics. Mol Cell Proteomics 1: 376-386, 2002. 
14. Chahrour O, Cobice D and Malone J: Stable isotope labelling methods in mass spectrometry-based quantitative proteomics. J Pharm Biomed Anal 113: 2-20, 2015.

15. Lu J, Tan M and Cai Q: The warburg effect in tumor progression: Mitochondrial oxidative metabolism as an anti-metastasis mechanism. Cancer Lett 356: 156-164, 2015.

16. National Research Council (US) Committee for the Update of the Guide for the Care and Use of Laboratory Animals. 8th edition. National Academies Press (US), Washington (DC), 2011.

17. Solaun MS, Mendoza L, De Luca M, Gutierrez V, López MP, Olaso E, Lee Sim BK and Vidal-Vanaclocha F: Endostatin inhibits murine colon carcinoma sinusoidal-type metastases by preferential targeting of hepatic sinusoidal endothelium Hepatology 35: 1104-1116, 2002.

18. Osinalde N, Sánchez-Quiles V, Akimov V, Blagoev B and Kratchmarova I: SILAC-based quantification of changes in protein tyrosine phosphorylation induced by interleukin-2 (IL-2) and IL-15 in T-lymphocytes. Data Brief 5: 53-58, 2015.

19. Osinalde N, Mitxelena J, Sánchez-Quiles V, Akimov V, Aloria K, Arizmendi JM, Zubiaga AM, Blagoev B and Kratchmarova I: Nuclear phosphoproteomic screen uncovers ACLY as mediator of IL-2-induced proliferation of $\mathrm{CD}^{+} \mathrm{T}$ lymphocytes. Mol Cell Proteomics 15: 2076-2092, 2016.

20. Liu X, Qiao B, Zhao T, Hu F, Lam AK and Tao Q: Sox2 promotes tumor aggressiveness and epithelialmesenchymal transition in tongue squamous cell carcinoma. Int J Mol Med 42: 1418-1426, 2018.

21. Hieda M, Matsuura N and Kimura H: Histone modifications associated with cancer cell migration and invasion. Methods Mol Biol 1238: 301-317, 2015.

22. Worley MJ Jr, Liu S, Hua Y, Kwok JS, Samuel A, Hou L, Shoni M, Lu S, Sandberg EM, Keryan A, et al: Molecular changes in endometriosis-associated ovarian clear cell carcinoma. Eur J Cancer 51: 1831-1842, 2015.

23. Lim W, Ryu S, Bazer FW, Kim SM and Song G: Chrysin attenuates progression of ovarian cancer cells by regulating signaling cascades and mitochondrial dysfunction. J Cell Physiol 233: 3129-3140, 2018.

24. Boland ML, Chourasia AH and Macleod KF: Mitochondrial dysfunction in cancer. Front Oncol 3: 292, 2013.

25. Ishikawa K, Takenaga K, Akimoto M, Koshikawa N, Yamaguchi A, Imanishi H, Nakada K, Honma Y and Hayashi J: ROS-generating mitochondrial DNA mutations can regulate tumor cell metastasis. Science 320: 661-664, 2008.
26. Ishikawa K, Koshikawa N, Takenaga K, Nakada K and Hayashi J: Reversible regulation of metastasis by ROS-generating mtDNA mutations. Mitochondrion 8: 339-344, 2008.

27. Esposito LA, Melov S, Panov A, Cottrell BA and Wallace DC: Mitochondrial disease in mouse results in increased oxidative stress. Proc Natl Acad Sci USA 96: 4820-4825, 1999.

28. Chazotte B: Labeling mitochondria with TMRM or TMRE. Cold Spring Harb Protoc 2011: 895-897, 2011.

29. Wood S Jr: Mechanisms of establishment of tumor metastases. Pathobiol Annu 1: 281-308, 1971.

30. Vaiopoulos AG, Athanasoula $\mathrm{KCh}$ and Papavassiliou AG: Epigenetic modifications in colorectal cancer: Molecular insights and therapeutic challenges. Biochim Biophys Acta 1842: 971-980, 2014.

31. Chen S and Chiu SK: AP4 activates cell migration and EMT mediated by 553 in MDA-MB-231 breast carcinoma cells. Mol Cell Biochem 407: 57-68, 2015.

32. Bae WK, Yoo KH, Lee JS, Kim Y, Chung IJ, Park MH, Yoon JH, Furth PA and Hennighausen L: The methyltransferase EZH2 is not required for mammary cancer development, although high EZH2 and low H3K27me3 correlate with poor prognosis of ER-positive breast cancers. Mol Carcinog 54: 1172-1180, 2015.

33. Yoo KH and Hennighausen L: EZH2 methyltransferase and H3K27 methylation in breast cancer. Int J Biol Sci 8: 59-65, 2012.

34. Warburg O: On the origin of cancer cells. Science 123: 309-314, 1956.

35. Ngo DC, Ververis K, Tortorella SM and Karagiannis TC: Introduction to the molecular basis of cancer metabolism and the warburg effect. Mol Biol Rep 42: 819-823, 2015.

36. Pelicano H, Xu RH, Du M, Feng L, Sasaki R, Carew JS, Hu Y, Ramdas L, Hu L, Keating MJ, et al: Mitochondrial respiration defects in cancer cells cause activation of akt survival pathway through a redox-mediated mechanism. J Cell Biol 175: 913-923, 2006.

37. Justus CR, Sanderlin EJ and Yang LV: Molecular connections between cancer cell metabolism and the tumor microenvironment. Int J Mol Sci 16: 11055-11086, 2015.

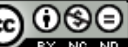

This work is licensed under a Creative Commons Attribution-NonCommercial-NoDerivatives 4.0 International (CC BY-NC-ND 4.0) License. 